Supplement of The Cryosphere, 15, 1889-1905, 2021

https://doi.org/10.5194/tc-15-1889-2021-supplement

(C) Author(s) 2021. CC BY 4.0 License.

(c) (i)

Supplement of

\title{
On the attribution of industrial-era glacier mass loss to anthropogenic climate change
}

Gerard H. Roe et al.

Correspondence to: Gerard H. Roe (groe@uw.edu)

The copyright of individual parts of the supplement might differ from the article licence. 
On the attribution of industrial-era glacier mass loss to anthropogenic climate change:

Supplementary material.

S1. Alternative synthetic little ice ages.
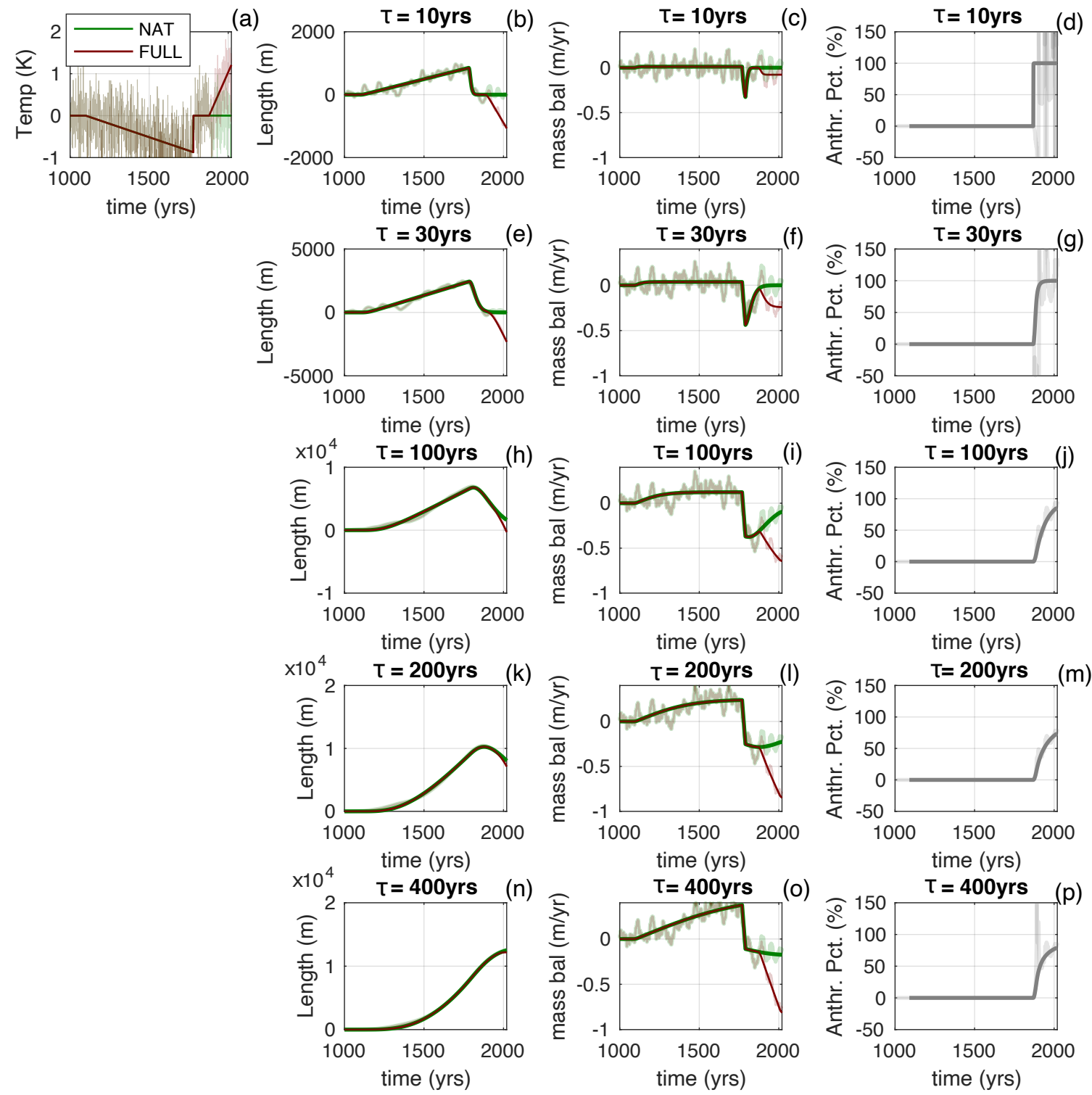

Figure S1. Same as Fig. 2, but with an extreme, unrealistic, LIA cooling ending in 1750. 

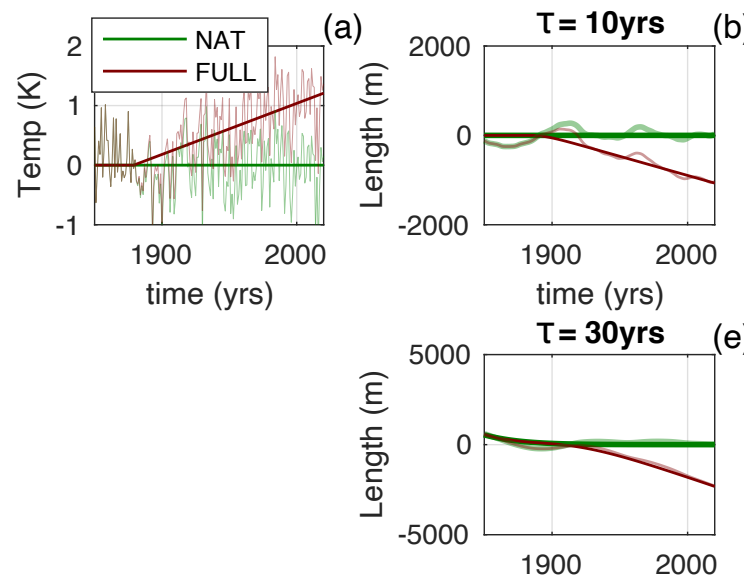

time (yrs)

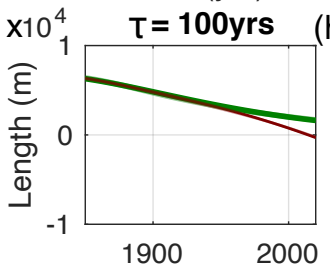

time (yrs)

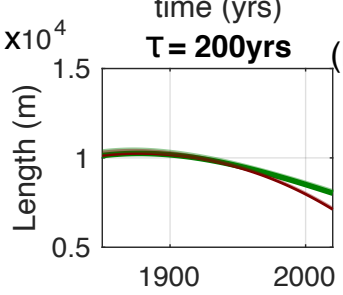

time (yrs)

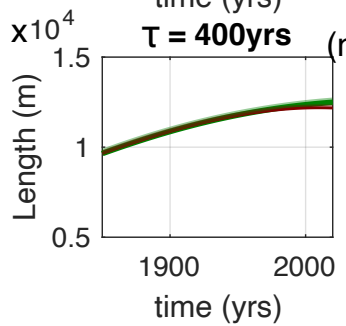

(b)

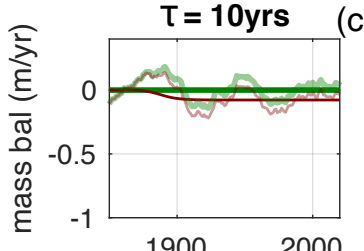

time (yrs)

$\mathrm{T}=30 \mathrm{yrs}$

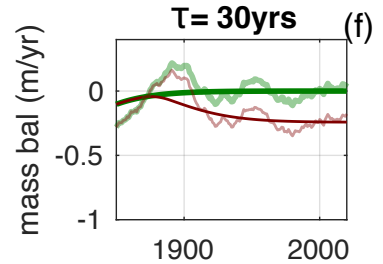

time (yrs)

$\mathrm{T}=100 \mathrm{yrs}$

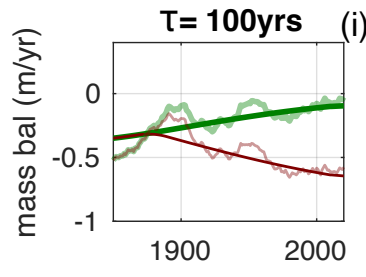

time (yrs)
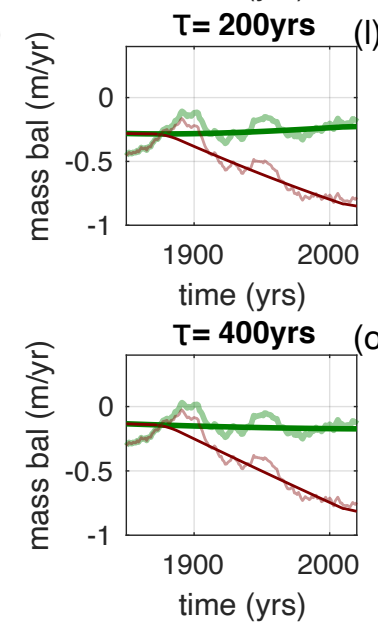

(c)

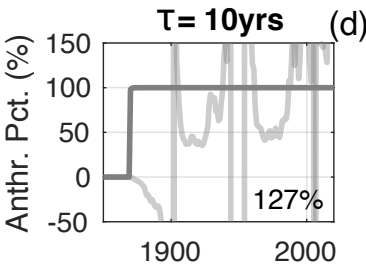

time (yrs)

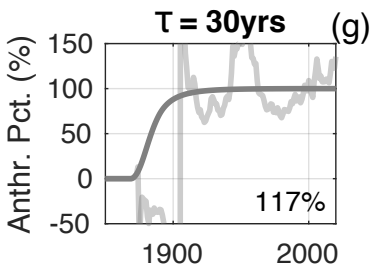

time (yrs)
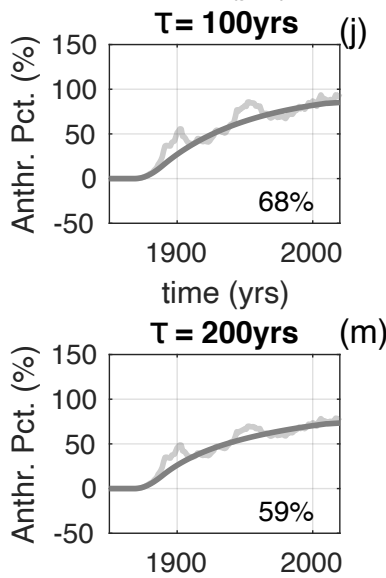

time (yrs)

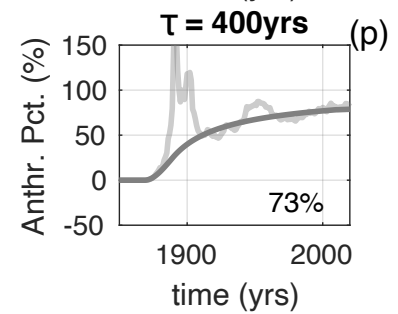

Figure S2. Same unrealistic LIA as Fig. S1, but zoomed into the period 1850 to 2020 . The percentages inserted into the right-hand columns give the anthropogenic cumulative mass loss relative to the total cumulative mass loss between 1880 and 2020 . Note these cumulative percentages will not, in general, be equal to the time average of $F_{a n t h}$. 

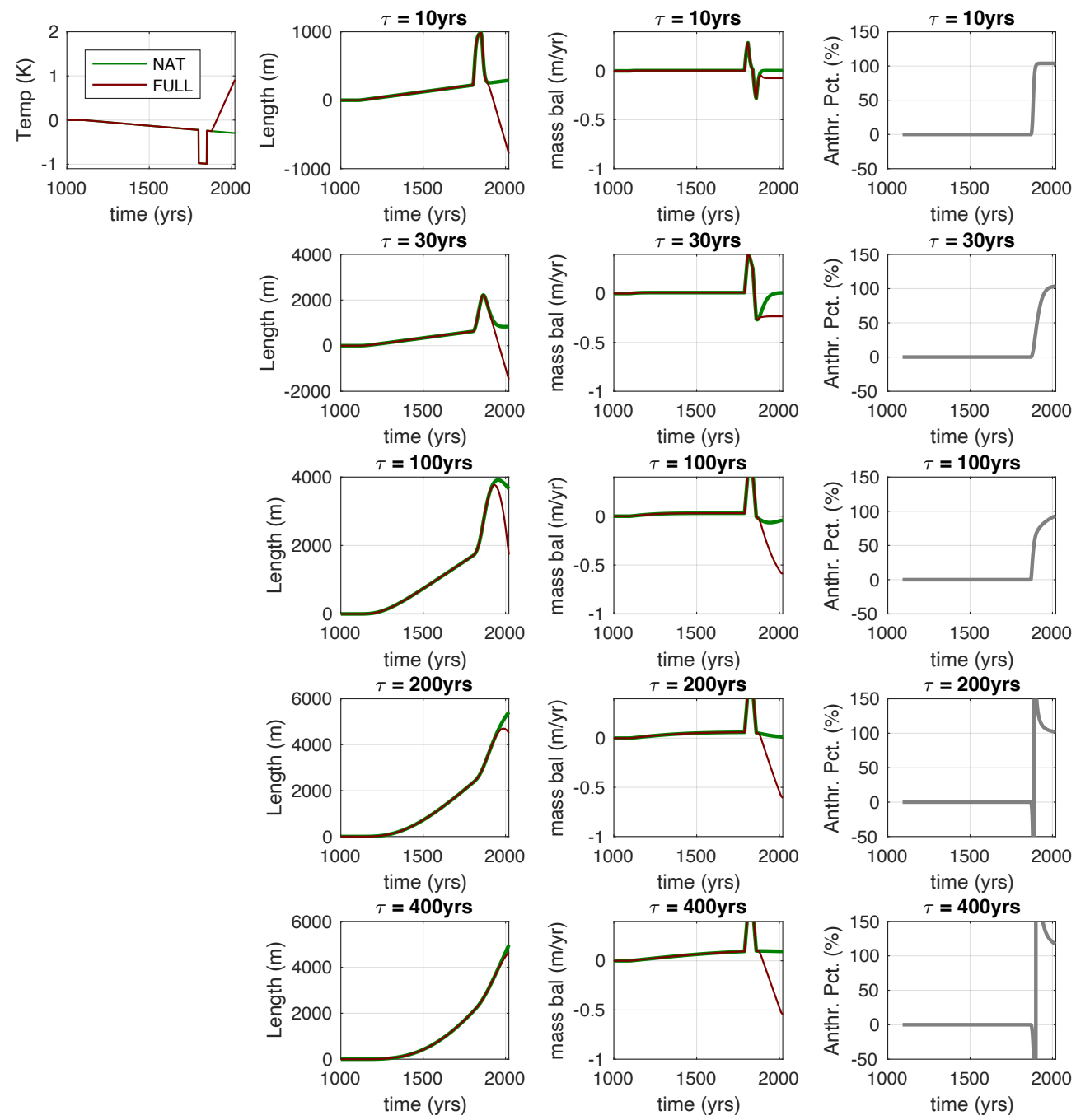

Figure S3. Long-term cooling plus a fifty-year top-hat cold event $\left(0.75^{\circ} \mathrm{C}\right)$ from 1800 to 1850 . This is intended to represent an unrealistically large cold event, perhaps associated with a local Little Ice Age. 

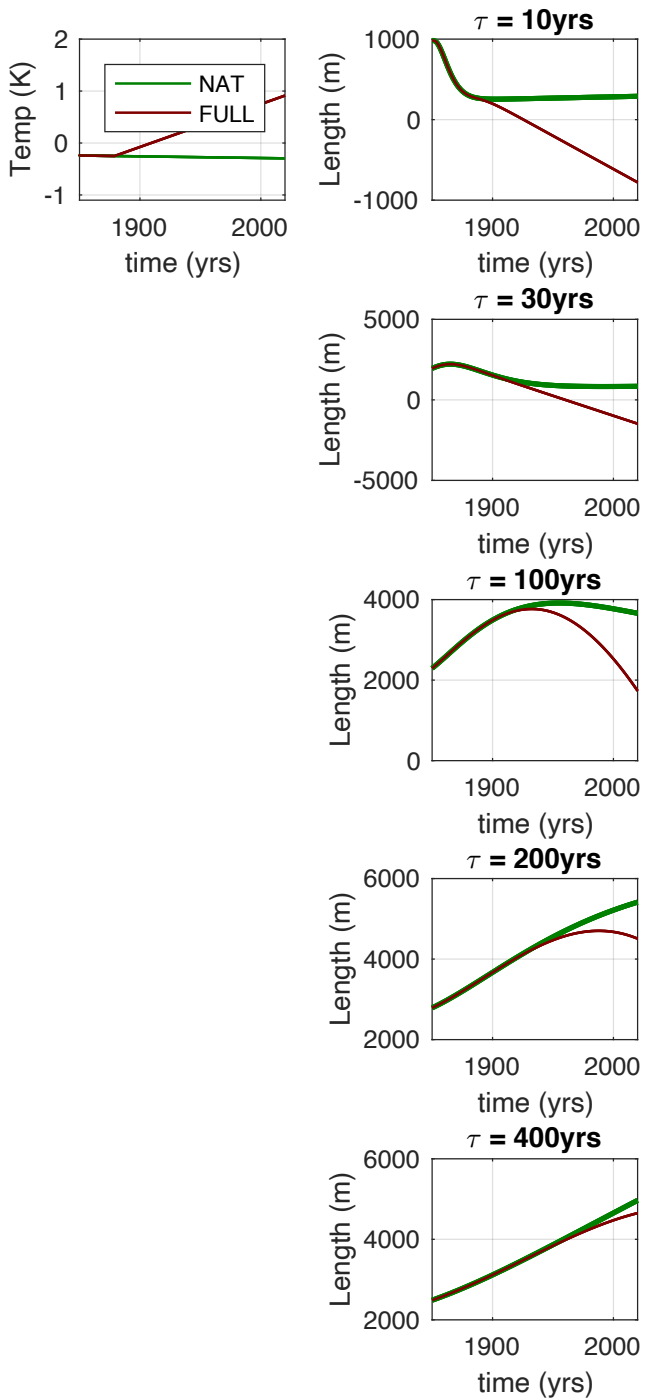
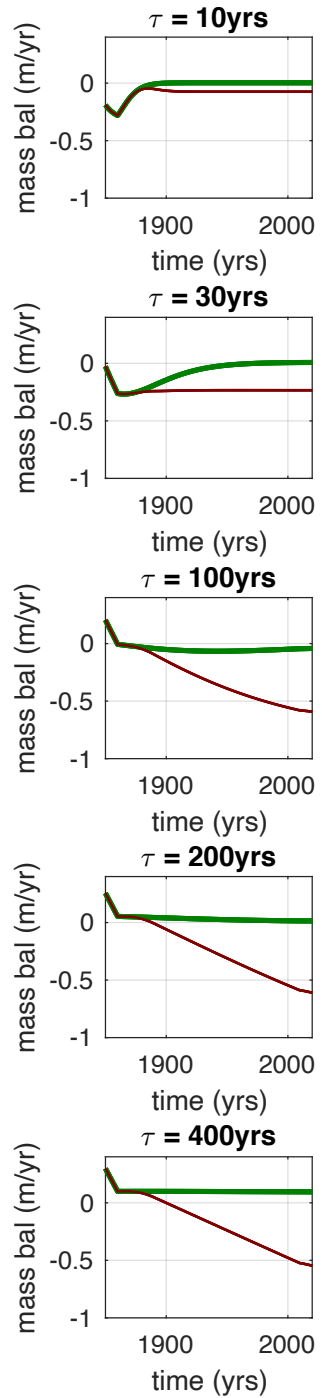
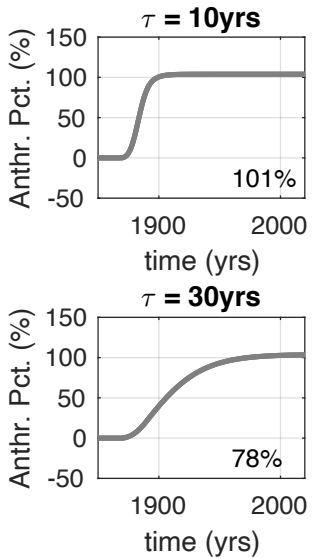

$=100 y r s$
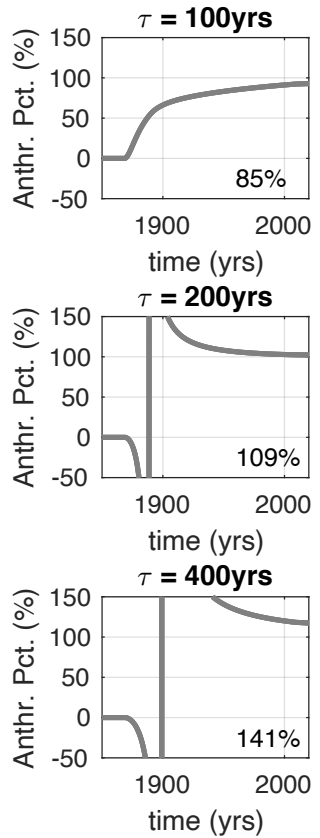

Figure S4. Same as Fig. S3, but for 1850 to 2020 . The percentages inserted into the right-hand columns give the anthropogenic cumulative mass loss relative to the total cumulative mass loss between 1880 and 2020. Note these cumulative percentages will not, in general, be equal to the time average of $F_{\text {anth }}$. 


\section{S2. Evaluation of linear approximation.}

Here we evaluate the impact of retaining the quadratic term in the mass loss: $b^{\prime}\left(\bar{L}+L^{\prime}(t)\right)$. Errors in cumulative mass loss

20 from simply integrating the linear term will vary by glacier, depending on how much of its preindustrial area it has lost (i.e., $L^{\prime}(t)$ cf. $\bar{L}$ ). They will be greatest for glaciers that have nearly vanished (or have already); however, this is not the norm in the global collection of length records (e.g., Leclercq et al., 2014). Many of the largest glaciers have retreated a small amount compared to their total area, in which case integrating $b^{\prime}$ is a good approximation.

25 We demonstrate this with two limiting cases. In Fig. S5, we show a small glacier with $\tau=33$ yr subject to a gradual millennial cooling, and then rapid anthropogenic warming (in the FULL) case, which causes the glacier to lose nearly all its area by 2020 (panel (b)). Panel (c) shows the yearly volume change, which is $b^{\prime} w \bar{L}$ for the linear approximation (dashed) and $b^{\prime} w\left(\bar{L}+L^{\prime}\right)$ including the evolving area. We assume a constant width $w$. In this case, the absolute volume changes become small as the glacier area shrinks. The cumulative loss is shown in panel (d), and is overestimated by the linear approximation. In reality,

30 the total volume is weighted towards the response to early forcing when the glacier was bigger. However, this has no effect on the anthropogenic fraction of cumulative mass loss (panel (e)), because there is no natural component of mass loss in this scenario.

Accounting for the changing glacier area affects cumulative loss if a large preindustrial disequilibrium is assumed. Figure S6

35 shows the same analysis, but for a case similar to Figs. 4 and 5 (main text), where the long cooling anomaly naturally ends in 1850. In this case, $F_{\text {anth }}$ for cumulative volume loss rises more slowly when the changing glacier area is taken into account (panel e). Thus, the linear approximation would overestimate $F_{\text {anth }}$. However, S7 shows the other limiting case, for a large glacier where $L^{\prime}$ is small compared to $\bar{L}$. Here, there is very little error associated with the linear approximation and essentially no effect on $F_{\text {anth }}$ for cumulative loss.

40

We expect that the linear approximation captures the basic evolution $F_{\text {anth }}$ for cumulative mass loss for the purposes of these analyses. We emphasize again that these issues also only affect conclusions about fractional attribution if a large preindustrial disequilibrium is assumed (Fig. S6). As discussed in the main text, such an assumption is not consistent with estimates of regional-to-global climate history over the last few centuries. Global assessments of total mass loss and contributions to sea

45 level would need to take the evolving glacier geometry into account. However, for the question of attribution of mass loss since 1850 , the analyses throughout this study show that the assumed climate history is clearly the more important issue. 
a)

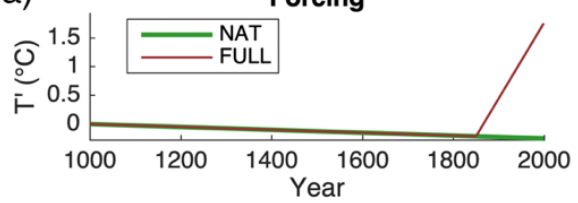

b)

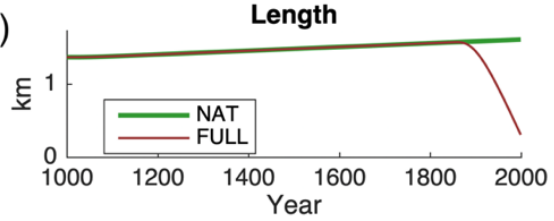

d)

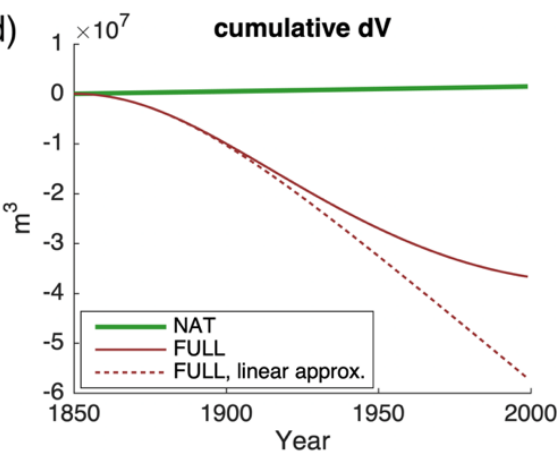

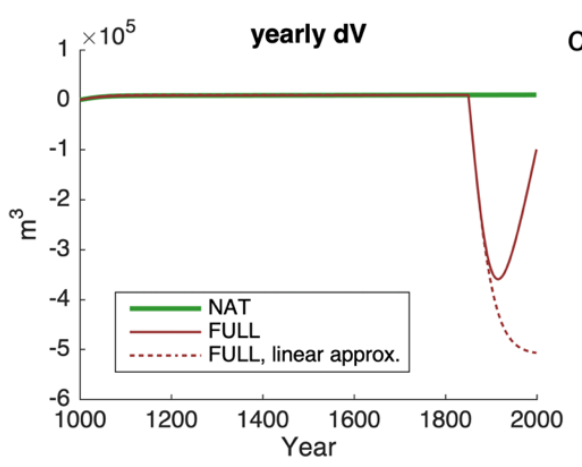

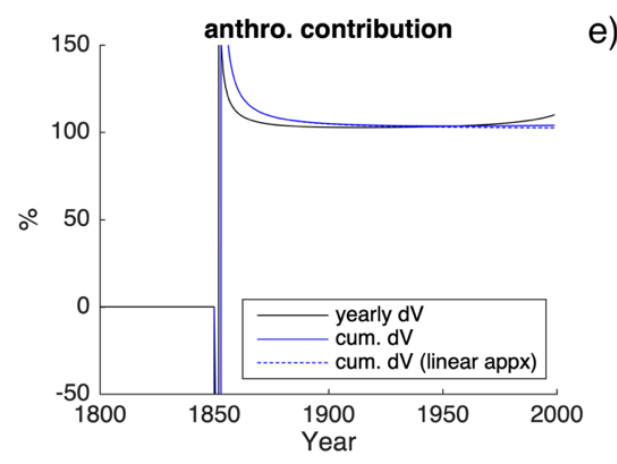

50 Figure S5. A limiting case where geometry changes drastically, challenging the linear approximation for cumulative mass loss. The glacier has $\tau=33$ yr. (a) Idealized gradual natural cooling and rapid anthropogenic warming. (b) Length response; the glacier nearly vanishes in the FULL case, which reduces the total volume losses associated with negative mass balance. (c) Yearly volume loss calculated by $b^{\prime}\left(\bar{L}+L^{\prime}\right)$ (solid lines) vs. $b^{\prime} \bar{L}$ (dashed). (d) Cumulative mass loss, which is overestimated by the linear approximation (dashed). e) Anthropogenic fraction for yearly and cumulative mass loss. This is unaffected by the approximation,

55 because there is no natural component of mass loss in this scenario. 
a)

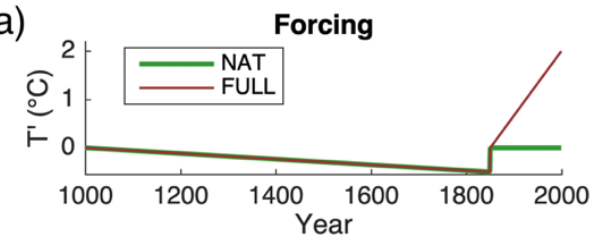

b)

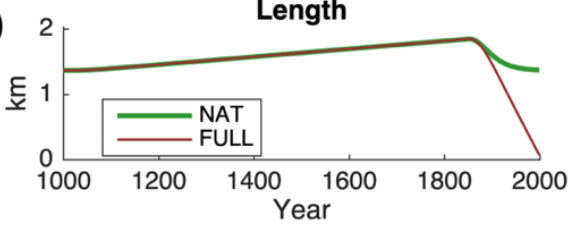

d)

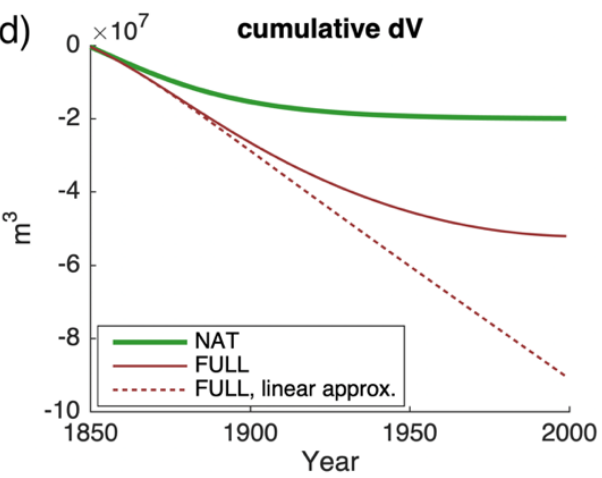

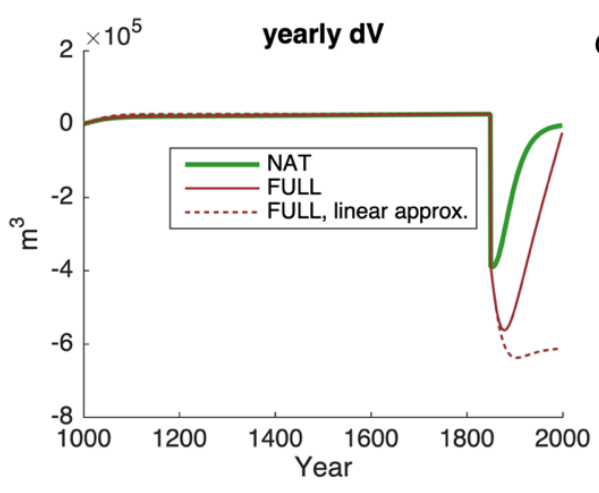

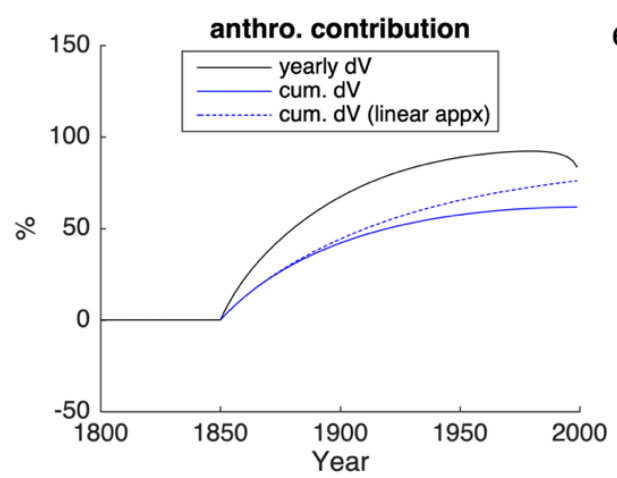

c)

e)

Figure S6. Same as S5 but for a scenario where the natural cooling trend abruptly ends in $\mathbf{1 8 5 0}$. Again, cumulative mass loss is lessened by the vanishing glacier area. In this case, the linear approximation also overestimates the anthropogenic mass loss

60 compared to total mass loss, because later mass loss (predominantly anthropogenic) should in reality be weighted less. Note that the anthropogenic fraction of yearly mass loss begins to dip down again as the glacier vanishes. This is due to the tiny residual imbalance in the counterfactual NAT case, which becomes a larger proportion of NAT/FULL as the glacier area shrinks to zero in the FULL case. 
a)

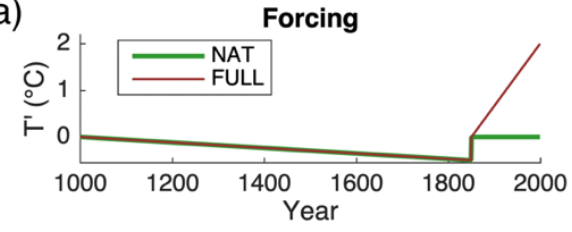

b)
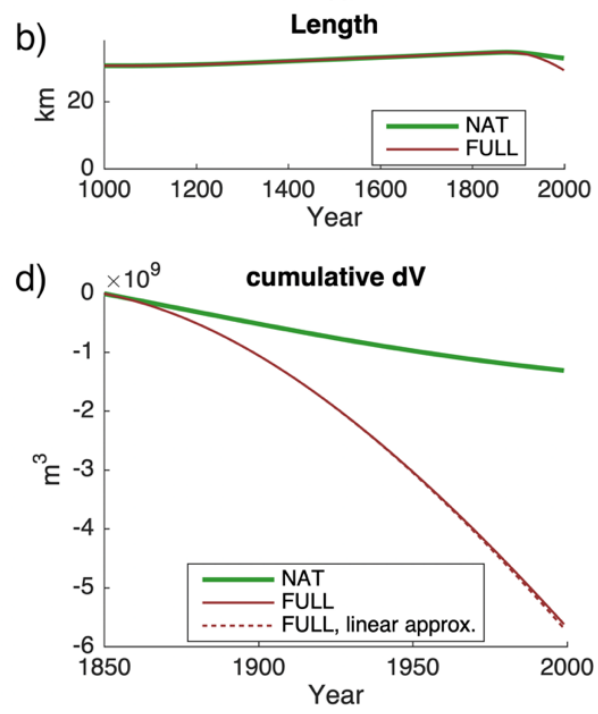
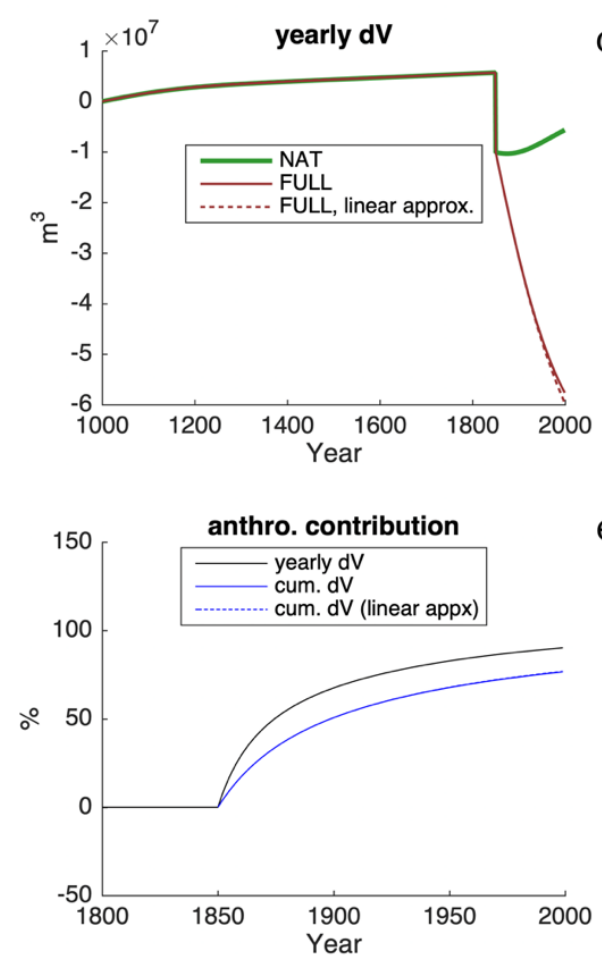

c)

e)

Figure S7. Same as Fig. S6 except for a very large glacier with $\tau=100 \mathrm{yr}$, whose retreat is small compared to its total area. In this case, the linear approximation for cumulative mass loss holds up well (d), and does not affect the anthropogenic mass loss. As described in the main text, $F_{\text {anth }}$ is less than $100 \%$ because of the assumed natural mass imbalance in 1850 , which is slow to diminish due to the 100-year response time. 

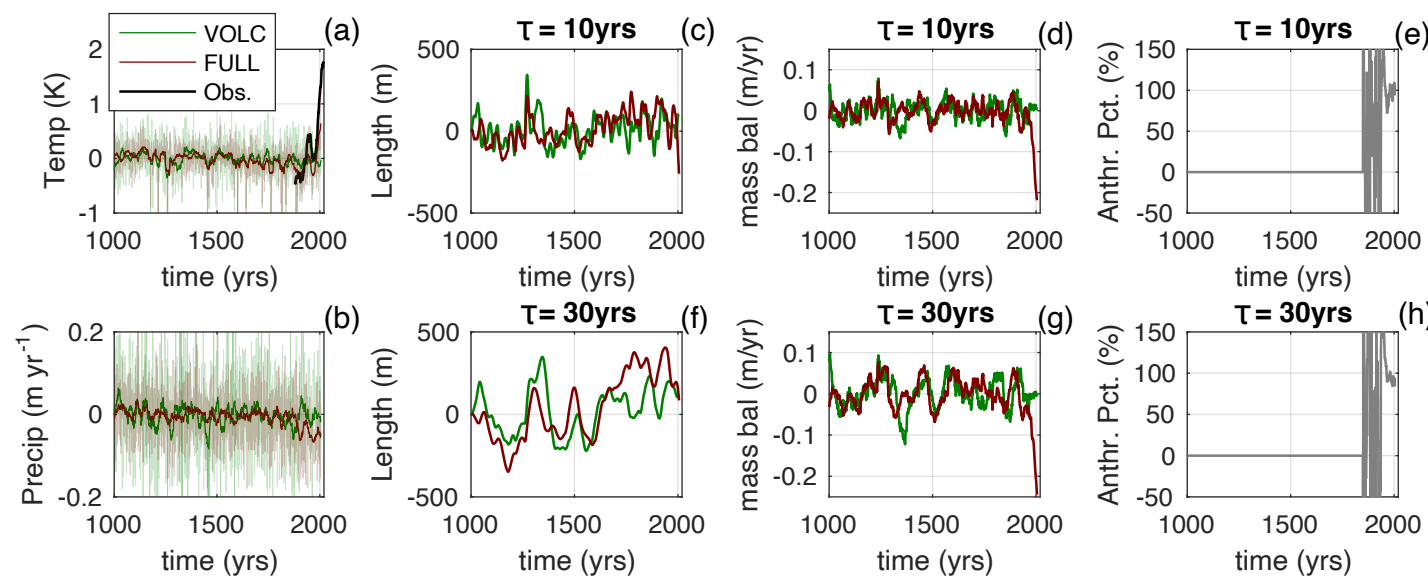

(b) $500 \quad \tau=30 y r s \quad$ (f)
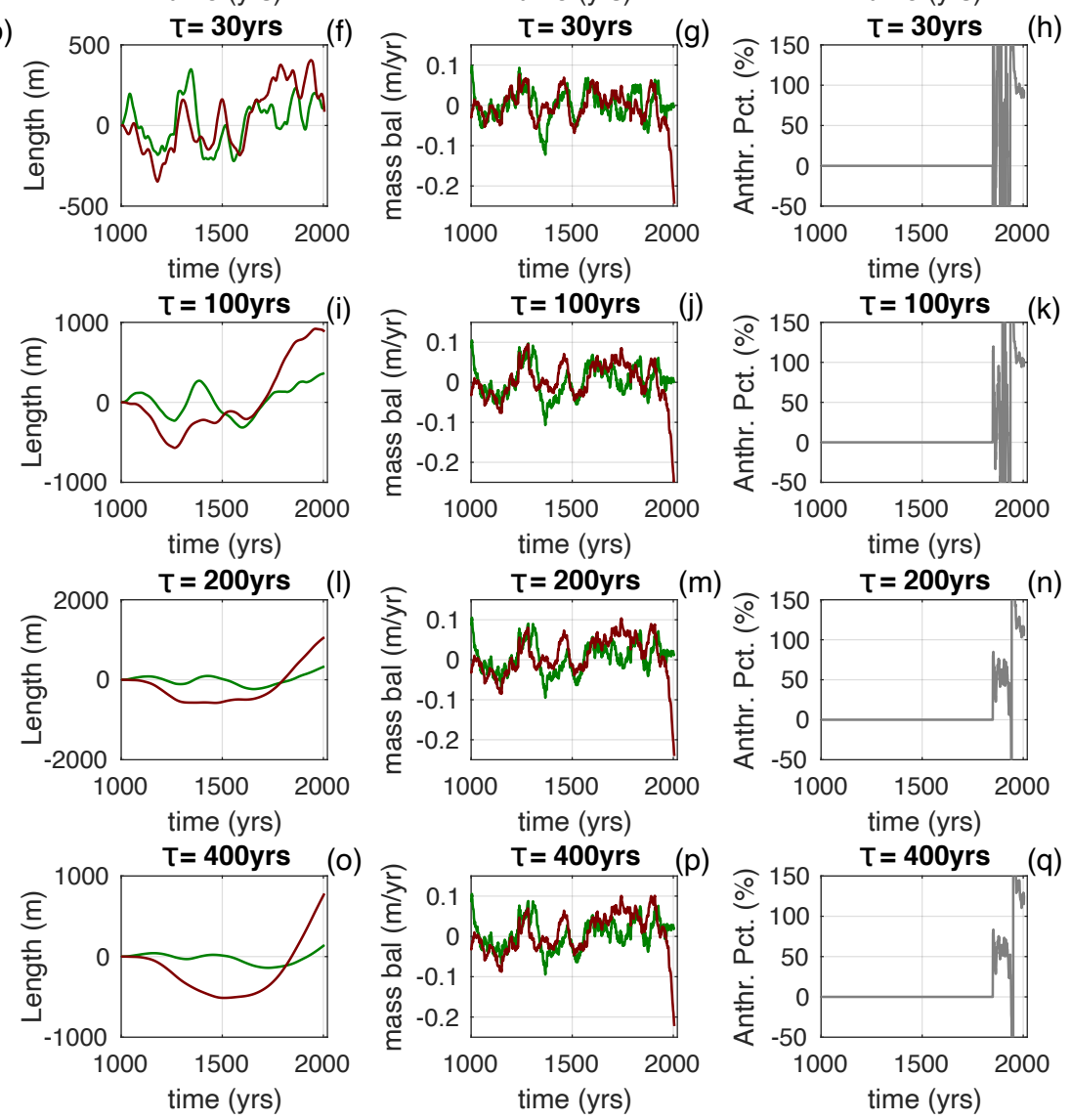

Figure S8. As for Fig. 7, but for a local point in the European Alps (47N, 10E). The CESM underestimates the observed warming between 1880 and 2020 , but still shows strongly negative mass balance with anthropogenic forcing included. 

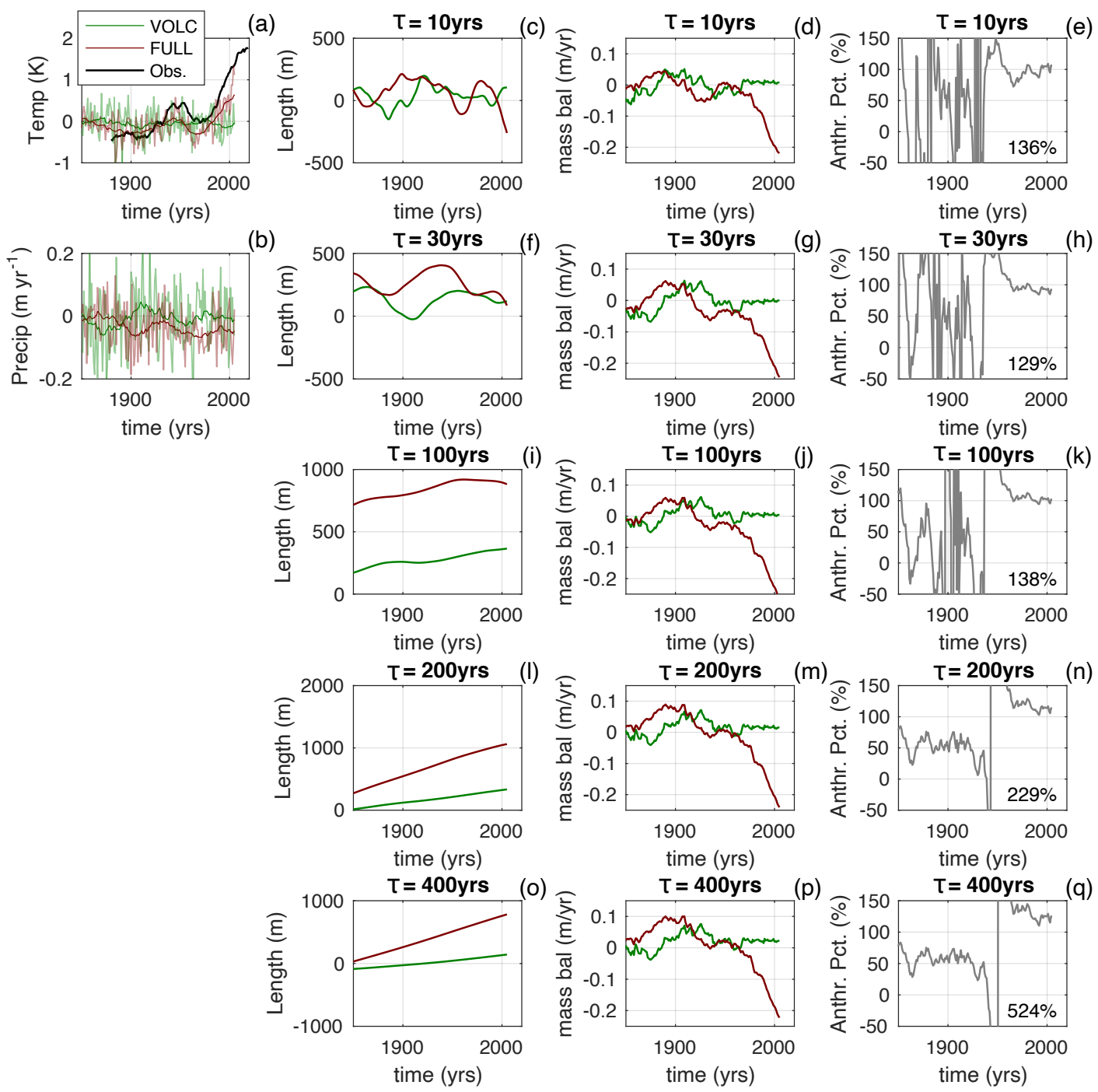

Figure S9. As for Fig. S8, but zoomed in to the period 1850 to 2020. The CESM underestimates the observed warming, but still shows strongly negative mass balance with anthropogenic forcing included. The percentages inserted into the right-hand columns give the anthropogenic cumulative mass loss relative to the total cumulative mass loss between 1880 and 2020 . Note these cumulative percentages will not, in general, be equal to the time average of $F_{\text {anth }}$. The $\tau=400 \mathrm{yr}$ case shows a large anthropogenic cumulative mass-loss fraction because the CESM model has cooler temperatures extending into the 1950s, unlike the observations. Hence the cumulative mass loss implied by the CESM output in the FULLcase is small. 

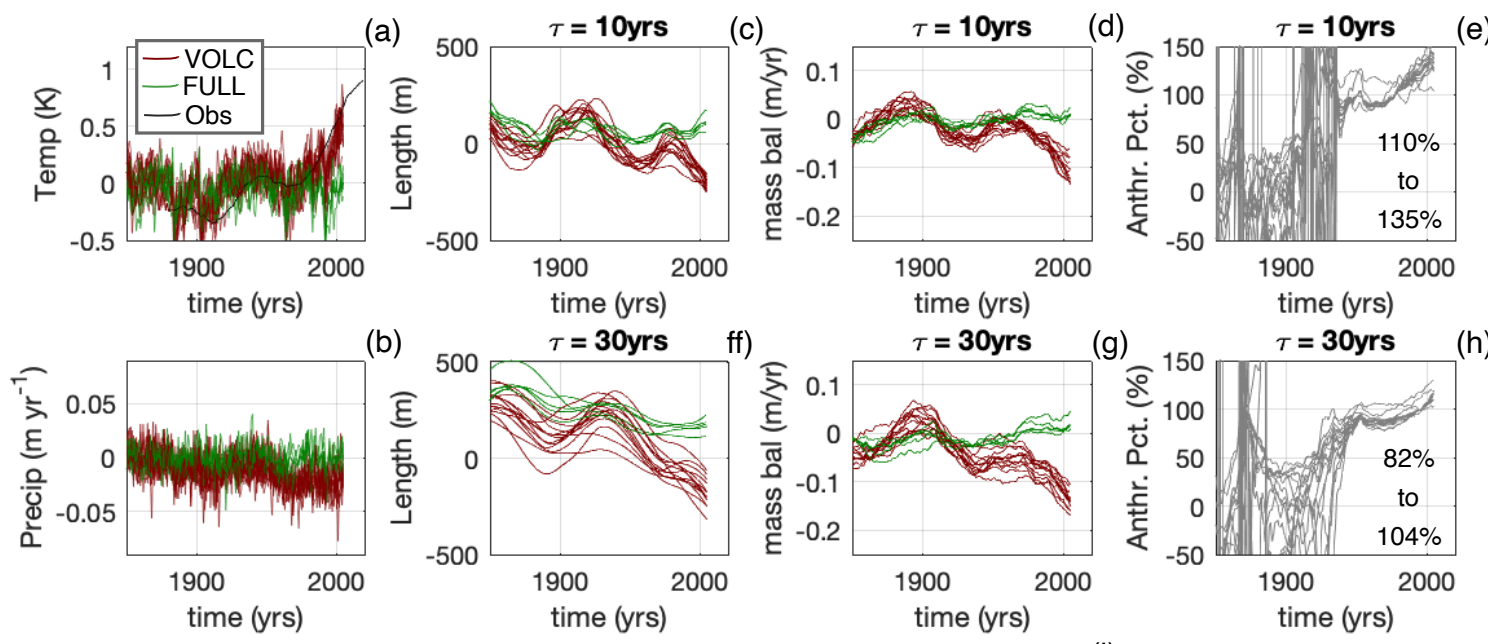

(b) $500 \quad \tau=30 y r s \quad f f)$
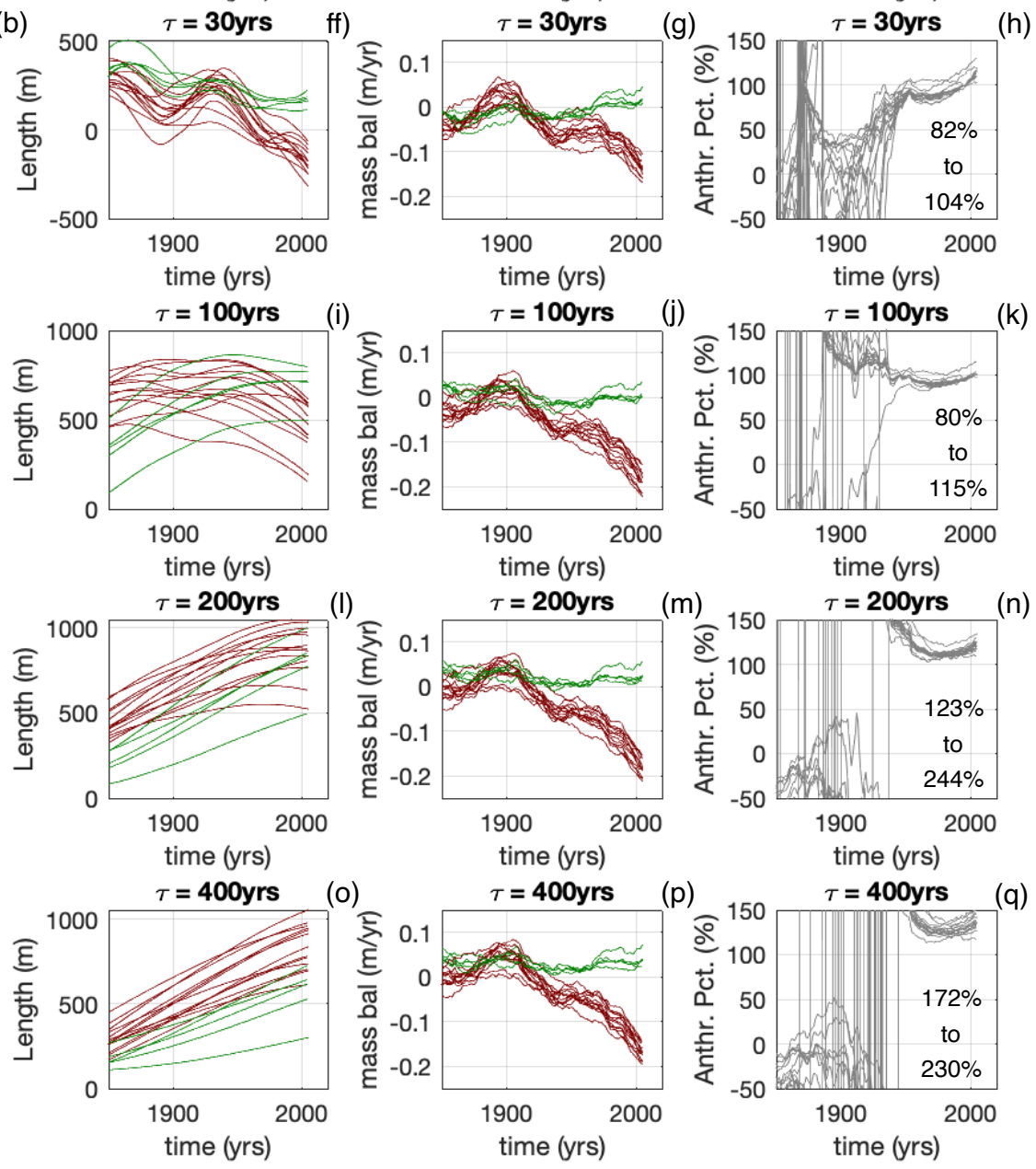

Figure S10. Same as Figure 8 but for individual CESM ensemble members. The FULL curves (13 members) are shown in red, and the natural variability ( 4 members) are shown in green. The percentages inserted into the right-hand columns are the full ranges among ensemble members for the anthropogenic cumulative mass loss relative to the total cumulative mass loss between 1880 and 2020. 


\section{S5: Relative importance of precipitation and temperature trends.}

85 On the millennium timescale, precipitation trends in climate models are small (e.g., Fig. 5, S11), and mostly consistent with white noise of internal, interannual variability (Parsons et al., 2017). Precipitation variability also has lower spatial coherence, meaning it will tend to average out at global scales. Environmental proxies provide some indication that more low-frequency variability in precipitation exists than in models (Dee et al., 2017). However, for the most part, precipitation acts as a noise maker for glacier mass balance, and there is no reason to suppose it would lead to widespread mass imbalance on centennial

90 time scales. On scales where climate variability is spatially coherent, mass-balance variability and glacier-length variability will also be coherent. In the global average much of this regional climate variability cancels out; and the response of massbalance and length to the global-scale external climate forcing will be more prominent (Huston et al., in press.)

We relate mass-balance forcing to temperature and precipitation (Roe and Baker, 2014):

$$
\beta b_{f}^{\prime}=\beta P^{\prime}-\alpha T^{\prime},
$$

95 where $\beta=\bar{L} / H$, and $\alpha=\mu L_{T>0} / H ; \mu$ is a melt-factor (which we take to be $0.65 \mathrm{~m} \mathrm{yr}^{-1} \mathrm{~K}^{-1}$ ), and $L_{T>0}$ is the length of the equilibrium glacier that experiences some melting during the year. From Eq. (A1), the precipitation anomaly equivalent to a temperature anomaly is:

$$
P^{\prime}=-\frac{\alpha}{\beta} T^{\prime}=-\mu \cdot \frac{L_{T>0}}{L_{t o t}} \cdot T^{\prime},
$$

Taking a value of $L_{T>0} / L_{t o t} \sim 0.8$, gives $P^{\prime} \sim 0.5\left[\mathrm{~m} \mathrm{yr}^{-1} \mathrm{~K}^{-1}\right] \cdot T^{\prime}$. In other words, a precipitation anomaly of $0.5 \mathrm{~m} \mathrm{yr}^{-1}$ is needed to offset a temperature anomaly of $1 \mathrm{~K}$. Roughly, in terms of interannual variability, a typical standard deviation in melt-season

100 temperature might be $\sim 0.5 \mathrm{~K}$ (Roe et al., 2017), and a typical standard deviation in annual precipitation is $\sim 15$ to $20 \%$ of the annual mean (say 0.5 to $4 \mathrm{~m} \mathrm{yr}^{-1}$, depending on the climatic setting). This means that, on interannual time scales, precipitation variability can be of comparable or greater importance than melt-season temperature for mass-balance variability (e.g., Medwedeff and Roe, 2017). However, whereas observed century-scale changes in temperature are $1 \mathrm{~K}$ or greater, precipitation changes are much smaller (a few percent per 1 K, e.g., Stocker et al., 2013). Thus, for the climate of the industrial-era, changes in temperature are generally much more important than changes in precipitation for affecting glacier mass balance. 